\title{
GIH-25 Prevalence and clinical spectrum of gastroesophageal reflux disease in Chinese population-a population based study
}

WM Wong, SK Lam, WM Hui, KC Lai, WHC Hu, *KF Lam, CLK Lam, NYH Wong, HHX Xia, AOO Chan, BCY Wong. Department of Medicine and *Department of Statistics and Actuarial Science, University of Hong Kong

Background and aims: Population-based data of gastroesophageal reflux disease (GERD) in Chinese population are lacking. The aim of this study is to study the prevalence and clinical spectrum of GERD and its complications in Chinese population and the effects of co-existing anxiety and depression on health care utilization.

Methods: 3605 ethnic Chinese households, randomly selected by a computer-assisted telephone interviewing system were invited to participate in a telephone survey using a validated GERD questionnaire and the Hospital Anxiety-Depression Scale (HAD). GERD was defined as heartburn and/or acid regurgitation.

Results: 2209 subjects (58\% female, mean age of 40.3) completed the interview (response rate 61\%). The annual, monthly and weekly prevalence of GERD symptoms were $29.8 \%, 8.9 \%$ and $2.5 \%$ respectively. Sex, age and socio-economic status were similar between subject with GERD and subjects without. By multiple logistic regression analysis, GERD symptoms were associated with NCCP (OR 2.3, 95\% CI 1.7-3.1), dyspepsia (OR 1.9, 95\% CI 1.4-2.5), globus (OR 1.8, 95\% CI 1.2-2.7), acid feeling in stomach (OR 5.8, 95\% CI 4.5-7.5) and NSAID intake (OR 2.3, 95\% CI 1.5-3.6), but not with dysphagia, bronchitis, asthma, hoarseness, pneumonia and history of smoking, alcohol, coffee, tea and aspirin intake. GERD patients had a significantly higher mean anxiety and depression score, and required more sick-leave when compared to subjects without. The frequency of heartburn $(\mathrm{P}<0.001)$, severity of acid regurgitation $(\mathrm{P}<0.001)$, female sex $(\mathrm{P}<0.001)$, higher socio-economic status $(\mathrm{P}<0.004)$ and the degree of anxiety $(\mathrm{P}<0.001)$ were independent factors associated with health seeking behaviour in GERD patients by multiple logistic regression analysis.

Conclusion: The prevalence of GERD was considerably lower than the western population. The frequency of heartburn, severity of acid regurgitation, female sex, higher socio-economic status and the degree of anxiety were independent factors associated with health care utilizations in GERD patients. 